\title{
The clinical effect of Embedding Therapy for Ramsay Hunt syndrome
}

\author{
Na Young Jo ${ }^{1}$, Jeong Du Roh ${ }^{1}$ \\ ${ }^{1}$ Dept. of Acupuncture \& Moxibustion Medicine, Je-Cheon Hospital of Traditional Korean Medicine, \\ Semyung University
}

\begin{abstract}
Objectives: The purpose of this study is to evaluate the clinical effects of Embedding Therapy for Ramsay Hunt syndrome.

Methods: 5 patients with Ramsay Hunt syndrome were treated with Embedding therapy. It was performed once a day, once a week. 15 20 Embedding threads were used in each Embedding treatment. The total number of Embedding therapy treatments was 4 or 8 . To evaluate the effects of Embedding therapy, we analyzed Yanagihara's score and House-Brackmann scale

Results: In case 1, After treatment the Yanagihara's score was changed from 3 to 7 and the House-Brackmann scale was changed from VI to V.

In case 2, After treatment the Yanagihara's score was changed from 12 to 33 and the House-Brackmann scale was changed from IV to I.

In case 3, After treatment the Yanagihara's score was changed from 3 to 9 and the House-Brackmann scale was changed from VI to $\mathrm{V}$.

In case 4, After treatment the Yanagihara's score was changed from 22 to 32 and the House-Brackmann scale was changed from III to I.

In case 5, After treatment the Yanagihara's score was changed from 23 to 37 and the House-Brackmann scale was changed from III to I.

Conclusion: Embedding Therapy is effective for improve the symptoms of Ramsay Hunt syndrom. Therefore, it will be used to treat Ramsay Hunt syndrome.
\end{abstract}

$\overline{\text { Key Words }}$ : Ramsay Hunt syndrome, Embedding Therapy, Facial palsy, Bell's palsy

\section{Introduction}

Ramsay Hunt syndrome is a viral disease accompanied with a shingles rash, ear pain and facial paralysis ${ }^{1)}$. The virus is mainly involved with geniculate ganglion but it rarely may affect auditory nerve, trigeminal nerve and IX, X, XI, XII cranial nerve. Thereby it can be symptoms of hypacusis, tinnitus, dizziness and trigeminal neuralgia ${ }^{2)}$. In general, the prognosis of Ramsay Hunt syndrome is not good as compared to the Bell's palsy). According to previous studies, the start of treatment has a significant effect on this disease recovery. Patients who treated early stage received a better remedial value than other patients ${ }^{4}$.

In Korean medicine, acupuncture, herbal medicine,

\footnotetext{
- Received : 23 May 2016 - Revised : 24 June 2016 Accepted : 24 June 2016

- Correspondence to : Roh Jeong-Du

Dept. of Acupuncture \& Moxibustion Medicine,

Je-Cheon Hospital of Traditional Korean Medicine, Semyung University

Semyungro 66, sinwoul-dong, jecheon city, chungbuk, 27136, Republic of Korea

Tel : +82-43-649-1816, Fax : +82-43-645-1382, E-mail : wsrohmio@hanmail.net
} 
physical therapy and taping therapy have been performed at Ramsay Hunt syndrome ${ }^{5)}$. In Korean medicine, Ramsay Hunt syndrome is within the scope of facial palsy. Various treatments have been attempted additionally, such as Hominis Placenta pharmacopuncture $^{6}$, electropuncture ${ }^{7)}$, scalp acupuncture therapy $^{8)}$, moxibustion ${ }^{9)}$ and Bee-Venom acupuncture ${ }^{10)}$. Recently, studies about embedding therapy have been reported with facial palsy ${ }^{11,12)}$. But various studies on the Ramsay Hunt syndrome treatment is insufficient.

Embedding Therapy is also known as Meridian point burial therapy or Medication thread burial therapy. The principle of this treatment that medication thread continue remain to under the skin so the effect lasts longer ${ }^{5)}$. Therefore, It is widely used from the beginning to the end stage of the facial palsy.

Kang's Embedding therapy study ${ }^{11)}$ was about sequela of facial palsy with one month later after onset, $\mathrm{Han}^{13)}$ reported the efficacy of Embedding therapy to facial palsy within one month after onset and Lee's Embedding therapy study ${ }^{12)}$ was about sequela of facial palsy with three months later after onset. But no study about Embedding therapy for Ramsay Hunt syndrome has ever been reported.

Ramsay Hunt syndrome is known that it is more difficult to recover than compared to Bell's palsy ${ }^{3)}$. Some patients suffered from complete facial palsy and severe facial or ear pain. Accordingly, we carried out Embedding therapy to Ramsay Hunt syndrome and report the results.

\section{Subject and Method}

\section{Subject}

The research involved 5 patients who hospitalized at the acupuncture \& moxibustion department of Semyung University Korean Medicine Hospital for Ramsay Hunt syndrome treatment from February 1,
2014 to December 31, 2014. They were diagnosed with Ramsay Hunt syndrome and they were treated at the Western medicine for initial treatment. they had no abnormalities with the brain CT or MRI. Purpose of the study, procedures and adverse reactions were explained enough to participants and all participants agreed to voluntarily participated.

Exclusion Criteria included the following:

(1) In association other disease showed up on the X-ray, CT or MRI.

(2) Patients with keloid, allergen or sensitive skin.

(3) Getting a skin laser treatment or going to be

(4) Patients with skin infections.

(5) Other patient that doctor determines not suitable for this study

\section{Treatment Method}

1) Embedding therapy

Medical thread for Embedding therapy were purchased from Dongbangchimgusa(Korea). The specifications of the used medical thread is $3 \mathrm{~cm}$, 29Gauge generalized smooth form. The medical procedure areas were based on the Park's ${ }^{14)}$ and Kim's ${ }^{15)}$ study. Frontalis muscle including the Yangbaek(GB14), Orbicularis oculi muscle, Levator labii superioris muscle, Zygomatic major muscle and Zygomatic minor including the Georyo (ST3), Jichang(ST4), Masseter muscle, Buccinator muscle including the Hyeopgeo (ST6) and Orbicularis Oris muscle are selected. Medical embedding thread was inserted in the same direction as the direction of muscle fiber in Frontalis muscle, Orbicularis oculi muscle, Levator labii superioris muscle, Zygomatic major muscle and Zygomatic minor and Orbicularis Oris muscle. Medical embedding thread was inserted in the vertical direction as the direction of muscle fiber in Masseter muscle and Buccinator muscle. All medical embedding were inserted outward from the facial center line and upward from below.

Embedding therapy was performed once a day, 
once a week. 15 20 Embedding thread were used in one time Embedding therapy. The number of medical embedding threads is different depending on degree of symptoms. The total number of Embedding therapy was 4 or 8 depending on the condition of the patient.

\section{2) Acupuncture treatment}

The acupuncture were disposable, stainless-steel filiform needles $(0.30 \mathrm{~mm}$ x $40 \mathrm{~mm})$ from Dongbang Acupuncture, Inc. Following the meridian points, the acupuncture was operated at the Yepung(TE17), Gakson(TE20), Nosik(TE19), Gyeonjeong (GB21) of the affected side before Embedding therapy. The acupuncture's retaining times were $15 \mathrm{~min}$.

\section{3) Herbal medicine}

Patients were taking the herbal medicine three times a day. The first week after admission were taking the ligigeopoongsan. Then for three weeks, patients were taking the Bogigeopoongsan. Starting five weeks were taking the Boheotang. Prescription medications were little different according to the characteristics of the patient.

\section{Investigation analytical method}

In order to the general characteristics of patients and the effects of Embedding therapy, we investigated Yanagihara's score ${ }^{16)}$ of five rating before and after treatment, 6) House-Brackmann scale ${ }^{17)}$ before and after treatment,

\section{Case reports}

\section{Case 1}

1. Name : Lee OO

2. Sex/Age : $M / 63$

3. Chief complain and current medical history

He complained Rt. facial palsy, Ear pain. He was a farmer. At that time of onset, he was exhausted by excessive farming. Patients visits a nearby hospital as soon as the disease outbreak. He was diagnosed as a Ramsay Hunt syndrome and prescribed the drugs. However, the disease was gradually progress and facial palsy was carried out to complete grade in two days. We could hardly observe facial muscle movement of the patient when he first visited our hospital.

4. Onset: 2014. 2. 24

5. Hospitalization period

2014. 2. 26 - 2014. 4. 4

6. Past medical history

He has no special medical history.

7. Family's medical history

He has no special family's medical history.

8. Change of Yanagihara's score

After treatment in case 1 the Yanagihara's score was changed from 3 to 7.(Table. 1.)

\begin{tabular}{|c|c|c|c|c|}
\hline Date & $3 / 9$ & $3 / 16$ & $3 / 23$ & $3 / 30$ \\
\hline score & 3 & 4 & 6 & 7 \\
\hline
\end{tabular}

9. Change of House-Brackmann scale

After treatment in case 1 the House-Brackmann scale was changed from VI to V.(Table. 2.)

\begin{tabular}{|c|c|c|c|c|}
\hline Date & $3 / 9$ & $3 / 16$ & $3 / 23$ & $3 / 30$ \\
\hline scale & VI & VI & V & V \\
\hline
\end{tabular}

\section{Case 2}

1. Name : Wang OO

2. Sex/Age : F/79

3. Chief complain and current medical history She complained Rt. facial palsy. The disease occurs without special motivation. Patients visits a nearby ear-nose-and-throat hospital as soon as the disease outbreak. She was diagnosed as a Ramsay Hunt syndrome and prescribed the drugs. However, the disease was gradually progress even the day. She was admitted immediately to the western medical hospital for 10 days. She received physical therapy and medications during hospitalization. Her symptoms during hospitalization 
were not substantially improved. We could observe weak facial muscle movement of the patient when she first visited our hospital.

4. Onset: 2014. 5. 29

5. Hospitalization period 2014. 6. 9 - 2014. 7. 2

6. Past medical history

She underwent surgery for uterine cancer in 1994. For the past thirty years she takes medicine to lower hypertension.

7. Family's medical history

She has no special family's medical history.

8. Change of Yanagihara's score

After treatment in case 2 the Yanagihara's score was changed from 12 to 33. (Table. 3.)

\begin{tabular}{|c|c|c|c|c|}
\hline Date & $6 / 10$ & $6 / 17$ & $6 / 24$ & $7 / 1$ \\
\hline score & 12 & 22 & 28 & 33 \\
\hline
\end{tabular}

9. Change of House-Brackmann scale

After treatment in case 2 the House-Brackmann scale was changed from IV to I.(Table. 4.)

\begin{tabular}{|c|c|c|c|c|}
\hline Date & $6 / 10$ & $6 / 17$ & $6 / 24$ & $7 / 1$ \\
\hline scale & IV & III & II & I \\
\hline
\end{tabular}

\section{Case 3}

1. Name : Lee OO

2. Sex/Age : M/19

3. Chief complain and current medical history

He complained Rt. facial palsy, Ear and facial pain, headache. He was a college student. At that time of onset, he was exhausted by excessive studying. Patients visits a nearby Korean clinic as soon as the disease outbreak. Korean medical doctor was invited to visit the hospital for more precise diagnosis. The disease was gradually progress and facial palsy was carried out to complete grade in four days. He visited the western medical hospital after five days from onset. He was diagnosed as a Ramsay Hunt syndrome and prescribed the drugs. We could hardly observe facial muscle movement of the patient when he first visited our hospital.

4. Onset: 2014. 7. 11

5. Hospitalization period

2014. 7. 18 - 2014. 9. 5

6. Past medical history

He has no special medical history.

7. Family's medical history

He has no special family's medical history.

8. Change of Yanagihara's score

After treatment in case 3 the Yanagihara's score was changed from 3 to 9.(Table. 5.)

\begin{tabular}{|c|c|c|c|c|c|c|c|c|}
\hline Date & $7 / 22$ & $7 / 29$ & $8 / 5$ & $8 / 12$ & $8 / 19$ & $8 / 26$ & $9 / 2$ & $9 / 9$ \\
\hline score & 3 & 3 & 7 & 7 & 7 & 9 & 9 & 9 \\
\hline
\end{tabular}

9. Change of House-Brackmann scale

After treatment in case 3 the House-Brackmann scale was changed from VI to V.(Table. 6.)

\begin{tabular}{|c|c|c|c|c|c|c|c|c|}
\hline Date & $7 / 22$ & $7 / 29$ & $8 / 5$ & $8 / 12$ & $8 / 19$ & $8 / 26$ & $9 / 2$ & $9 / 9$ \\
\hline scale & VI & VI & V & V & V & V & V & V \\
\hline
\end{tabular}

\section{Case 4}

1. Name : Sim OO

2. Sex/Age : F/63

3. Chief complain and current medical history She complained Lt. facial palsy. The disease occurs no special motivation. Patients visits a nearby ear-nose-and-throat clinic as soon as the disease outbreak. She was diagnosed as a Ramsay Hunt syndrome and prescribed the drugs. However, the disease was gradually progress. She was admitted to the western medical hospital for a month. She received physical therapy and medications during hospitalization. Her symptoms had improved a little during hospitalization. She had continued therapy in outpatient department after discharge for 5 month but the symptoms did not change. We could observe facial muscle movement of the patient when she first visited our hospital but the move was incomplete state.

4. Onset: 2014. 1. 16

5. Hospitalization period 
2014. 8. 28 - 2014. 10. 8

6. Past medical history

She underwent surgery for chronic degenerative arthritis of both knee in 2012 .

7. Family's medical history

Her mother was diagnosed cerebral infarction.

8. Change of Yanagihara's score

After treatment in case 4 the Yanagihara's score was changed from 22 to 32.(Table. 7.)

\begin{tabular}{|c|c|c|c|c|c|c|c|c|}
\hline Date & $9 / 9$ & $9 / 16$ & $9 / 23$ & $9 / 30$ & $10 / 7$ & $10 / 14$ & $10 / 21$ & $10 / 28$ \\
\hline score & 22 & 22 & 24 & 27 & 28 & 30 & 32 & 32 \\
\hline
\end{tabular}

9. Change of House-Brackmann scale

After treatment in case 4 the House-Brackmann scale was changed from III to I.(Table. 8.)

\begin{tabular}{|c|c|c|c|c|c|c|c|c|}
\hline Date & $7 / 22$ & $7 / 29$ & $8 / 5$ & $8 / 12$ & $8 / 19$ & $8 / 26$ & $9 / 2$ & $9 / 9$ \\
\hline scale & III & III & III & II & II & II & I & I \\
\hline
\end{tabular}

\section{Case 5}

1. Name : Kim OO

2. Sex/Age : F/72

3. Chief complain and current medical history

She complained Rt. facial palsy. The disease occurs no special motivation. Patients visits a nearby Korean clinic as soon as the disease outbreak. However, the symptoms was little change. She visited the western medical hospital She was diagnosed as a Ramsay Hunt syndrome and prescribed the drugs. She visited our hospital to receive Korean medical treatment. We could observe weak facial muscle movement of the patient when she first visited our hospital.

4. Onset: 2014. 11. 17

5. Hospitalization period 2014. 11. 19 - 2014. 12. 19

6. Past medical history

She underwent surgery for uterine myoma in 1974. For the past thirty years she takes medicine to lower hypertension and hyperglycemia.

7. Family's medical history
Her brother died of complications from diabetes

8. Change of Yanagihara's score

After treatment in case 2 the Yanagihara's score was changed from 23 to 37.(Table. 9.)

\begin{tabular}{|c|c|c|c|c|}
\hline Date & $11 / 24$ & $12 / 1$ & $12 / 8$ & $12 / 15$ \\
\hline score & 23 & 26 & 34 & 37 \\
\hline
\end{tabular}

9. Change of House-Brackmann scale

After treatment in case 2 the House-Brackmann scale was changed from III to I.(Table. 10.)

\begin{tabular}{|c|c|c|c|c|}
\hline Date & $11 / 24$ & $12 / 1$ & $12 / 8$ & $12 / 15$ \\
\hline scale & III & III & II & I \\
\hline
\end{tabular}

\section{Discussion}

Ramsay Hunt syndrome and Bell's palsy both show symptoms of facial paralysis. Both are called Guanwasa in Korean medicine. The facial nerve is the seventh cranial nerve. It controls the same side of the face muscles. It related to taste, tears and saliva secretion, but it consists mostly of motor nerve fibers ${ }^{15}$. So patients undergoes a facial movement disorder.

Embedding Therapy is to be embedded melting thread by using a specially designed apparatus. That melting thread also used as the material for surgical sutures for surgery ${ }^{18)}$.

Embedding Therapy has the principle that analogous to placing acupuncture for a period of times. It can make consistently effect on acupuncture points until melt away ${ }^{19)}$.

Medication thread to stay under the skin and it cause of stimulate the resisting power. It widely used in chronic diseases, musculoskeletal disorders, dermatology disorders and gynecological disorders in korean medicine ${ }^{14)}$.

In recent years Embedding therapy has been used in the field of plastic surgery. In Hong's study explains the principles of treatment ${ }^{19)}$ and in other studies research on its effectiveness ${ }^{20)}$. Embedding 
therapy is mainly used in the field of facial plastic ${ }^{21)}$. In the same vein, researches have been reported about facial paralysis.

In Park's study ${ }^{14)}$, Embedding Therapy can be used in facial paralysis. In addition, Kang's ${ }^{11)}$ study, Lee's ${ }^{12)}$ study, Hong's ${ }^{19)}$ study and Han's ${ }^{13)}$ study has proceed in clinical research.

Depending on the results of previous research, we tried to improve symptoms of Ramsay Hunt syndrome through treatment with Embedding Therapy.

The purpose of Embedding therapy on facial palsy is to restore of facial movement, prevention of strain and promote of nerve regeneration. Scale that can accurately grasp the degree of paralysis in order to objectively evaluate the effect of treatment is necessary. Yanagihara's score and House-Brackmann scale are most often used for evaluate of facial palsy $^{22)}$.

In case 1, His symptoms were rapidly deteriorating. Symptoms took only two days to reach complete facial palsy. During hospitalization in our hospital the symptoms are hardly improving. After treatment the Yanagihara's score was changed from 3 to 7 and the House-Brackmann scale was changed from VI to $\mathrm{V}$.

In case 2, She was hospitalized in western medical hospital as soon as occur symptoms. Her symptoms are stopped in House-Brackmann scale IV. Symptom changes were weak during first hospitalization. In parallel to the Embedding Therapy the symptoms were greatly improved. After treatment the Yanagihara's score was changed from 12 to 33 and the House-Brackmann scale was changed from IV to I.

In case 3, He almost did not get treatment nearly 5 days after onset. Meanwhile symptoms become very severe. His symptoms are reached in House -Brackmann scale VI. During hospitalization in our hospital the symptoms have been a little better. After treatment the Yanagihara's score was changed from 3 to 9 and the House-Brackmann scale was changed from VI to $\mathrm{V}$.

In case 4, She visited our hospital seven months later from outbreak. Her symptoms are stopped in House-Brackmann scale III. There was little change in symptoms for six months. In parallel to the Embedding Therapy the symptoms were improved. After treatment the Yanagihara's score was changed from 22 to 32 and the House-Brackmann scale was changed from III to I.

In case 5 , She immediately begin treatment and symptoms did not fast progresses. Her symptoms are stopped in House-Brackmann scale III. In parallel to the Embedding Therapy the symptoms were greatly improved. After treatment the Yanagihara's score was changed from 23 to 37 and the House-Brackmann scale was changed from III to I.

Although the number of cases in this study is too small, most of the cases have high therapeutic effects as compared to the previous studies. In Kim's study, the average score changes of Yanagihara's score for two weeks is about five ${ }^{23)}$. In this case, the average score changes of Yanagihara's score for two weeks is about seven. However this study included relatively a few cases, so the statistical analysis was difficult. There were no side effects after treatment, the patients were satisfied with the treatment. Judging from the results of this study, Embedding therapy about Ramsay Hunt syndrome may be a effective way and we hope that futher studies about Ramsay Hunt syndrome and Embedding therapy will be performed. This study will have the sufficient meanings as prior materials for further research on Embedding therapy about Ramsay Hunt syndrome.

\section{Conclusion}

Through our research, we obtained the following conclusions. In this case study, we have found that the Embedding therapy is to change the Yanagihara's score and House-Brackmann scale on Ramsay Hunt syndrome. The symptoms of all patients were 
improved. But the therapeutic effect was better about patients which have milder symptoms. Embedding therapy can be used as a treatment related to Ramsay Hunt syndrome. And it is worth to study in further research.

\section{References}

1. Back MK. Granular Cell Myoblastoma Larynx. 2ed rev. ed. Seoul:Iljogak. 1997:78.

2. Chu MJ, Yang SD, Shin SO. Analysis of the Prognostic Factors of Ramsay-Hunt Syndrome. J Korean Med Ophthalmol Otolaryngol Dermatol 1996;39(2):217-22.

3. Kim DJ, Kim JK, Kim JH. The Prognostic Factors of the Facial Nerve Paralysis in Herps Zoster Oticus. J Korean Med Ophthalmol Otolaryngol Dermatol. 1992;35(2):286-93.

4. Jung TK, Jeon HK, Lee KS, Yoon TH, Chu KC. Ramsay Hund Syndrome; Treatment of Facial Nerve palsy with Acyclovir and Prednisolone. J Korean Med Ophthalmol Otolaryngol Dermatol. 1995;38(6):842-6.

5. Korean Acupuncture \& Moxibustion Society. The acupuncture and moxibustion medicine. Pa-ju:Jipmoondang. 2012:200,625-9.

6. Lee EY, Yook TH, Kim EH, Lee JH, KimYH. The Clinical Observation of peripheral facial paralysis used Aqua-acupuncture treatment. The Journal of Korean Acupuncture \& Moxibustion Society. 2002;19(3):11-23.

7. Lee SW, Han SW. Clinical study of facial nerve paralysis through electro - acupunctre treatment. The Journal of Korean Acupuncture \& Moxibustion Society. 1999;16(4):149-63.

8. Choi YJ, Yoon KJ, Kim MS, Park JY, Jeon JC, Lee $\mathrm{TH}$, et al. Effects of Scalp Acupuncture with Usual Acupuncture on Peripheral Facial Palsy in Comparison with Usual Acupuncture Only. The $\mathrm{J}$ of Korean Acupuncture \& Moxibustion Society. 2010;27(6):101-9.
9. Kwon SJ, Song HS, Kim KH. The Influence of Moxibustion and Basic Compound Therapy on Peripheral Facial Paralysis. The J of Korean Acupuncture \& Moxibustion Society. 2000;17(4): 160-71.

10. Kim MS, Kim HJ, Park YJ, Kim EH, Lee EY. The clinical research of the efficacy of bee venom aqua-acupuncture on peripheral facial paralysis. The Journal of Korean Acupuncture \& Moxibustion Society. 2004; 21(4):251-62.

11. Kang EK, Kim JH, Seo HS. The Clinical investiation studies in peripheral facial paralysis using Needle-Embedding Therapy. The Journal of Korean Korean Medical Ophthalmology \& otolaryngology \& Dermatology. 2009;22(2): 118-27.

12. Lee CW, Lee SM, Jeon JH, Kim JI, Kim YI. Effects of Needle-Embedding Therapy on Sequelae of Peripheral Facial Palsy : A Case Series. The Journal of Korean Acupuncture \& Moxibustion Society. 2011;28(4):93-103.

13. Han JM, Yoon JW, Kang NR, Ko WS, Yoon HJ. The Clinical investigation studies in early stage of intractable peripheral facial paralysis using Needle-Embedding Therapy. The Journal of Korean Korean Medical Ophthalmology \& Otolaryngology \& Dermatology 2012;25(3): 113-128.

14. Park YU. The Thread-embedding Therapy. Seoul: Hanglimseowon. 2003:23,27-8.

15. Kim JH, Kwon HJ, Song JH, Choi DY, Lee SH, Lee JD. A Review of the Anatomy of Face for the Clinical Application of Facial Acupuncture. The Journal of Korean Acupuncture \& Moxibustion Society. 2008;25(3):221-8.

16. Yanagihara N. Grading of Facial Palsy. In Facial Nerve Surgery, Proceedings: Third International Symposium on Facial Nerve Surgery, Zurich, 1976. U. Fisch(Ed). Amstelveen, Netherlands: Kugler Medical Publications. Birmingham, Al: Aesculapius Publishing Co. 1977:533-5. 
17. Kang TS, Vrabec JT, Giddings $\mathrm{N}$ et al. Facial nerve grading systems(1985 2002): beyond the House-Brackmann scale. Otol Neurotol. 2002; 23(5):767-71.

18. Wen MS. Mai xian liao fa zhi bai bing. Beijing: Ren min jun yi chu ban she. 2002: 20-44.

19. Hong KE. Comprehension of Embedding Therapy Through Meridian Muscle System-Focused on Face. The Journal of Korean Acupuncture \& Moxibustion Society. 2008;25(3):215-9.

20. Lee KS, Ko MK, Lee JH, Kim MJ, Hong KE. The Effect of Facial Embedding Therapy on Skin Elasticity and Moisture Content. The Journal of Korean Acupuncture \& Moxibustion Society. 2011;28(5):111-9.
21. Lee JC, Park SH, Yoon JH, Kim JW, Lim CG. Preliminary Standard Procedure for Face Lift and Correction of Nasolabial Fold using Thread -Embedding (Maeseon) of Korean Medicine.The Journal of Korean Medical Ophthalmology \& Otolaryngology \& Dermatology 2013;26(4):43-50.

22. LEE WS, Woon BM, Kim DY, Lee JH. Clinical Application of FEMA Grading System. Korean J Otolaryngol. 1998;41(11):1378-82.

23. Kim HC, Kim JH, Kim YI. A Comparative Study on Improvement of Bell's Palsy and Ramsay Hunt Syndrome According to Treatment Duration. The Journal of Korean Acupuncture \& Moxibustion Society. 2008;25(4):31-9. 